\title{
Decentralized Nonlinear Model Predictive Control for 3D Formation of Multirotor Micro Aerial Vehicles with Relative Sensing and Estimation*
}

\author{
I. Kagan Erunsal ${ }^{1,2} \quad$ Alcherio Martinoli $^{2} \quad$ Rodrigo Ventura ${ }^{1}$
}

\begin{abstract}
Index Terms-Formation control, Decentralized Nonlinear Model Predictive Control (NMPC), Multirotor Micro Aerial Vehicles (MAVs), Pose-Graph Moving Horizon Estimation (PGMHE)

\section{INTRODUCTION}

In recent years, extensive research is conducted on the coordination and cooperation strategies of multirotor Micro Aerial Vehicles (MAVs) to perform high-level missions such as scientific exploration, search and rescue, intelligence gathering etc. [1], [2]. The main motivator for this interest is the fact that the deployment of multiple vehicles reduces the risk of mission failures and provides higher performance and flexibility through parallelism [3]. Among the main subproblems of cooperative control, formation control is usually an essential component and Model Predictive Control (MPC) is a promising tool to carry out this task deliberately. Since MPC is architecturally flexible and handles the performance and constraints systematically in parallel, it is drawing more attention nowadays [4]. Among MPC methods, especially Nonlinear Model Predictive Control (NMPC) is particularly suitable to control the robots whose fast dynamics are needed to be predicted by nonlinear models and constraints as in multirotor MAVs. Additionally, for large scale systems, Decentralized NMPC (D-NMPC) strategies are advantageous since they address the computational complexity by dividing the overall optimization problem into decoupled subproblems and by reducing communication requirements [4]. Furthermore, in order to deploy highly autonomous multirotor MAVs in non-trivial environments, several researchers focus on elaborating local and relative sensing in formation control and try to solve its limitations [5].

The intersection of multi-rotor MAVs, formation control and decentralized - distributed MPC is studied by several researchers in the literature [2], [6], [7], [8]. However, they either focus on the communication and global sensing or utilize linear MPC as methodology. Relative sensing in formation control of multi-rotor MAVs has also an important place in the literature [5], [9]. However, their work do not employ MPC framework. Although the paper [10] apply realtime receding-horizon optimization of trajectories that guarantee the rendezvous for only mobile robots, they presented a novel approach including relative sensing in local coordinate frames of the robots. For interested readers, other important references consist of [11], [12], [13], [14], [15].

In the view of presented literature, to the best of our knowledge, there is a literature gap for the $3 \mathrm{D}$ formation

*This work was partially supported by the FCT grant [PD/BD/135151/2017], the FCT doctoral program RBCog and the FCT project [UID/EEA/50009/2013].

${ }^{1}$ Institute for Systems and Robotics (ISR), IST, Lisbon, Portugal

${ }^{2}$ Distributed Intelligent Systems and Algorithms Laboratory (DISAL), EPFL, Lausanne, Switzerland

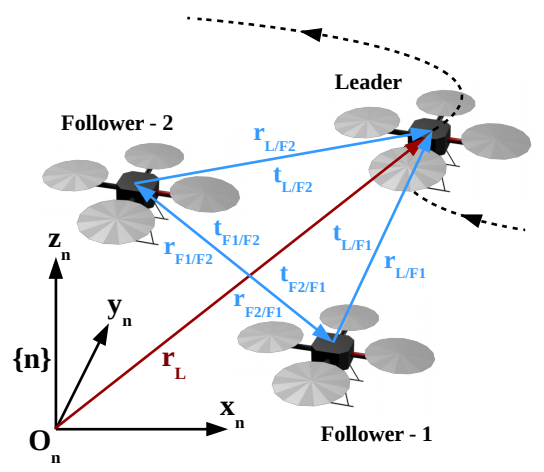

Fig. 1. Formation control problem with one leader and two followers, the inter-vehicle positions, angles and trajectory followed are also shown.

control problem of multi-rotor MAVs considering the intersection of relative sensing, formulation in local coordinate frames, D-NMPC structure without any explicit communication under sensor uncertainty. As a result, this paper aims this gap and provides the following contributions. First, it proposes a Decentralized NMPC framework using only sensing, particularly relative sensing, and estimation, diminishing the problems related to communication possessed by the other decentralized and distributed approaches. Second, It formulates the problem in individual local coordinate frames of the robots, similar to approach explained in [16] but in a decentralized setting. Third, it introduces a Pose-Graph Moving Horizon Estimator (PG-MHE) in order to obtain filtered and unknown states required by D-NMPC as the initial conditions of future predictions.

\section{Problem Definition}

In this work, the leader-follower type of formation strategy is selected due to its implementation simplicity and high performance. There is only one leader in the team equipped with global sensors or a localization unit similar to one in [17] that obtains global information for position and velocity tracking. This is a logical assumption because indoor global localization sensors are expensive and require high onboard computation capacity. In this respect, the leader can be seen as a decoupled agent from the formation subsystem and the trajectory tracking activity can be seen as a disturbance to formation control. The follower agents endowed exclusively with an on-board, limited FOV, 3D, relative localization system for measuring inter-vehicle positions and orientations of neighbour vehicles in terms of relative range, bearing and angle information for formation maintenance. A concrete implementation of a similar sensor can be found in [18]. Furthermore, all agents are equipped with an optic flowsonar sensor couple to obtain linear velocities and an IMU to acquire linear accelerations, rotational velocities and absolute roll and pitch information. Magnetometer cannot be 


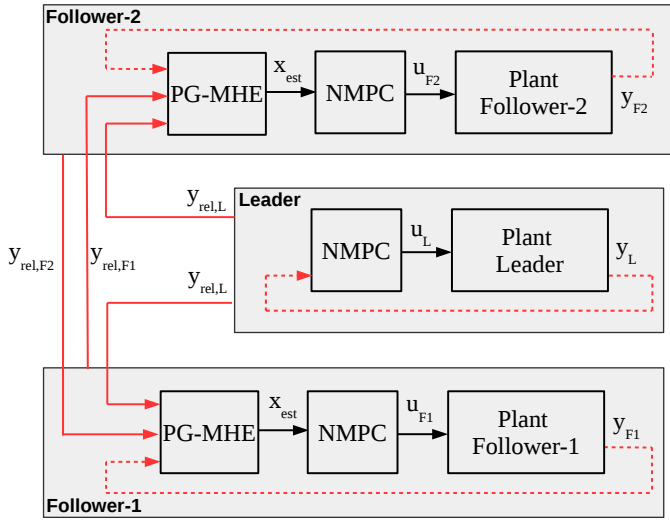

Fig. 2. Decentralized PG-MHE and MPC structure in formation control. accurately used in the problem because it is assumed that the vehicles operate inside of a building. This implies that the absolute yaw information can not be obtained. It is assumed that obstacles do not exist along the path. This assumption is made in order to simplify the problem. The overall aim of the group is tracking a trajectory while maintaining a prescribed formation. Each vehicle has its own onboard computer for NMPC computations. Since the approach proposed requires no communication, the issues like packet losses and delays are not considered. However, noise in all onboard sensors mentioned above exist. This scenario is depicted in Fig. 1.

\section{Methodology}

The decentralized control/estimation architecture is shown in Fig. 2. As can be seen from the figure, while each follower vehicle has one PG-MHE and one NMPC for formation control, the leader has one NMPC for trajectory tracking and it is decoupled from the formation subsystem. While the dashed-red lines show the sensed local variables, solid-red lines indicate sensed relative variables. In this decentralized structure, each follower utilizes only locally estimated/sensed variables. No communication is present in the architecture. In this figure, $u, x, y$ represent the input, state and output (raw sensor information), respectively. Furthermore, sensing-wise, the graph is undirected in formation subsystem, directed between leader and followers and complete as whole [19].

\section{A. Pose-Graph Moving Horizon Estimator}

We combine multiple measurements of the relative pose of the neighbours, with own motion sensing (from, e.g., optical flow sensor and IMU), in order to both filter out noise in relative pose measurements of each neighbour and to estimate its velocity with respect to an inertial frame. As in SLAM literature [20], we model this estimation problem using a factor graph of poses - a Pose Graph (PG) where the nodes are the variables to be estimated and the edges are factors, representing statistical dependencies among variables. The filter input is the sensor readings from the previous time steps, up to a temporal horizon, i.e., a Moving Horizon Estimator (MHE).

\section{B. Decentralized Nonlinear Model Predictive Control}

In each instance, NMPC constructs and solves an OCP by taking dynamics, constraints and controlled variables into account. A typical example is shown in (1) for a generic case [21].
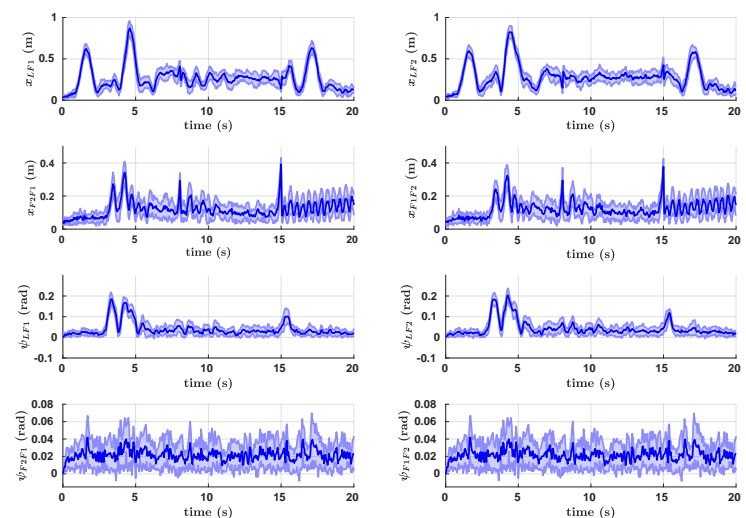

Fig. 3. The mean and standard deviations of error norms of relative positions and yaw angles between robots: L: Leader, F1: Follower-1, F2: Follower-2

$$
\begin{array}{cl}
\begin{array}{c}
\operatorname{minimize} \\
\boldsymbol{u}(0), \ldots, \boldsymbol{x}(N) \\
\text { minu }(N-1)
\end{array} & \sum_{k=0}^{N-1} c_{k}(\boldsymbol{x}(k), \boldsymbol{u}(k), \boldsymbol{r}(k))+c_{N}(\boldsymbol{x}(N), \boldsymbol{u}(N), \boldsymbol{r}(N)) \\
\text { subject to } & \boldsymbol{x}(k+1)=f_{k}(\boldsymbol{x}(k), \boldsymbol{u}(k)), \quad k=0, \ldots, N-1 \\
& g_{k}(\boldsymbol{x}(k), \boldsymbol{u}(k))=0, \quad k=0, \ldots, N-1 \\
& h_{k}(\boldsymbol{x}(k), \boldsymbol{u}(k)) \leq 0, \quad k=0, . ., N-1 \\
& g_{N}(\boldsymbol{x}(N))=0
\end{array}
$$

where $N$ is the selected horizon length, $\boldsymbol{x}(0), \ldots, \boldsymbol{x}(N)$, $\boldsymbol{u}(0), \ldots, \boldsymbol{u}(N)$ and $\boldsymbol{r}(0), \ldots, \boldsymbol{r}(N)$ represent the evolution of the state, input and reference respectively, $c_{k}$ 's are the stage cost functions, $c_{N}$ is the terminal cost function, $f_{k}$ 's are used to express nonlinear dynamic equality constraints, $g_{k}$ 's and $h_{k}$ 's denote other, possibly nonlinear, equality and inequality constraints respectively and $g_{N}$ stands for the terminal constraints. In this problem, the cost functions include the relative position and yaw errors between vehicles, non-gravitational inertial force and body fixed moments. The nonlinear dynamic equality constraints consist of the vehicle's own full dynamics and the neighbor vehicles' dynamics with constant linear and angular velocity assumption. Finally, other constraints are the upper and lower bounds of inputs and states, i.e. safety limits and physical limitations etc.

\section{Simulation AND Results}

The mathematical modeling, controller implementation, solver generation and simulation have been performed in MATLAB 2017a with Intel Core i7-7700HQ processor by using ACADO solver with modified RTI scheme [22], [23]. One leader and two followers are included. This scenario is designed in a way to include various test cases such as the formation control in linear motion, formation regeneration, formation control in spiral motion etc.

The error norms of the relative position vectors and yaw angles between the agents are shown in Fig. 3 for 10 runs. As can be seen, although the relative quantities between followers are quite low, $0.11 \mathrm{~m}$ and $0.03 \mathrm{rad}$ in average (steady-state), there are larger errors, $0.3 \mathrm{~m}$ and $0.05 \mathrm{rad}$ between leader and followers. This is because the followers behave very similarly and there are estimation errors of the leader due to constant velocity assumption. Note that these are the initial results and the important aspect here is to achieve convergence in all scenarios even if the assumptions (constant future velocities) do not hold for some trajectories. 


\section{REFERENCES}

[1] D. De Silva, "Formation Control for Unmanned Aerial Vehicles," Master's thesis, Instituto Superior Tecnico (IST), Portugal, 2012.

[2] A. Monteriù, A. Freddi, and S. Longhi, "Nonlinear decentralized model predictive control for unmanned vehicles moving in formation,' Information Technology and Control, vol. 44, no. 1, pp. 89-97, 2015.

[3] Z. Chao, L. Ming, Z. Shaolei, and Z. Wenguang, "Collision-free uav formation flight control based on nonlinear mpc," in 2011 international conference on electronics, communications and control (ICECC). IEEE, 2011, pp. 1951-1956.

[4] U. Eren, A. Prach, B. B. Koçer, S. V. Raković, E. Kayacan, and B. Açıkmeşe, "Model predictive control in aerospace systems: Current state and opportunities," Journal of Guidance, Control, and Dynamics, vol. 40, no. 7, pp. 1541-1566, 2017.

[5] F. Schiano, A. Franchi, D. Zelazo, and P. R. Giordano, "A rigiditybased decentralized bearing formation controller for groups of quadrotor uavs," in 2016 IEEE/RSJ International Conference on Intelligent Robots and Systems (IROS), Oct 2016, pp. 5099-5106.

[6] A. Bemporad and C. Rocchi, "Decentralized linear time-varying model predictive control of a formation of unmanned aerial vehicles," in Decision and Control and European Control Conference (CDC-ECC), 2011 50th IEEE Conference on. IEEE, 2011, pp. 7488-7493.

[7] R. Van Parys and G. Pipeleers, "Distributed MPC for multi-vehicle systems moving in formation," Robotics and Autonomous Systems, vol. 97, pp. 144-152, 2017.

[8] Q. Yuan, J. Zhan, and X. Li, "Outdoor flocking of quadcopter drones with decentralized model predictive control," ISA transactions, vol. 71, pp. 84-92, 2017.

[9] A. Franchi, P. Stegagno, and G. Oriolo, "Decentralized multi-robot encirclement of a $3 \mathrm{~d}$ target with guaranteed collision avoidance," Autonomous Robots, vol. 40, no. 2, pp. 245-265, 2016.

[10] S. Gowal and A. Martinoli, "Real-time optimization of trajectories that guarantee the rendezvous of mobile robots," in 2012 IEEE/RSJ International Conference on Intelligent Robots and Systems. IEEE, 2012, pp. 3518-3525.

[11] S. M. Huck, M. Rueppel, T. H. Summers, and J. Lygeros, "Rcopterxexperimental validation of a distributed leader-follower MPC approach on a miniature helicopter test bed," in 2014 European Control Conference (ECC). IEEE, 2014, pp. 802-807.

[12] A. T. Hafez, A. J. Marasco, S. N. Givigi, M. Iskandarani, S. Yousefi, and C. A. Rabbath, "Solving multi-uav dynamic encirclement via model predictive control," IEEE Transactions on Control Systems Technology, vol. 23, no. 6, pp. 2251-2265, 2015.

[13] H. Fukushima, K. Kon, and F. Matsuno, "Distributed model predictive control for multi-vehicle formation with collision avoidance constraints," in Decision and Control, 2005 and 2005 European Control Conference. CDC-ECC'05. 44th IEEE Conference on. IEEE, 2005, pp. 5480-5485.

[14] K. Yamamoto, K. Sekiguchi, and K. Nonaka, "Experimental verification of formation control by model predictive control considering collision avoidance in three dimensional space with quadcopters," in 2017 11th Asian Control Conference (ASCC), Dec 2017, pp. 16021607.

[15] Y. Kuwata, A. Richards, T. Schouwenaars, and J. P. How, "Distributed robust receding horizon control for multivehicle guidance," IEEE Transactions on Control Systems Technology, vol. 15, no. 4, pp. 627641, 2007.

[16] I. K. Erunsal, R. Ventura, and A. Martinoli, "Nonlinear model predictive control for $3 \mathrm{~d}$ formation of multirotor micro aerial vehicles with relative sensing in local coordinates," in International Conference on Intelligent Robots and Systems (submitted), arXiv: 1904.03742.

[17] M. Blsch, S. Weiss, D. Scaramuzza, and R. Siegwart, "Vision based mav navigation in unknown and unstructured environments," in 2010 IEEE International Conference on Robotics and Automation, May 2010, pp. 21-28.

[18] D. Dias, R. Ventura, P. Lima, and A. Martinoli, "On-board visionbased 3d relative localization system for multiple quadrotors," in 2016 IEEE International Conference on Robotics and Automation (ICRA), May 2016, pp. 1181-1187.

[19] K.-K. Oh, M.-C. Park, and H.-S. Ahn, "A survey of multi-agent formation control," Automatica, vol. 53, pp. 424-440, 2015.

[20] M. Kaess, H. Johannsson, R. Roberts, V. Ila, J. J. Leonard, and F. Dellaert, "isam2: Incremental smoothing and mapping using the bayes tree," The International Journal of Robotics Research, vol. 31, no. 2, pp. 216-235, 2012.

[21] Acado toolkit, nonlinear model predictive control and moving horizon estimation. [Online]. Available: acado.github.io/cgt_overview.html
[22] B. Houska, H. Ferreau, and M. Diehl, "ACADO Toolkit - An Open Source Framework for Automatic Control and Dynamic Optimization,' Optimal Control Applications and Methods, vol. 32, no. 3, pp. 298312, 2011.

[23] M. Kamel, M. Burri, and R. Siegwart, "Linear vs nonlinear MPC for trajectory tracking applied to rotary wing micro aerial vehicles,' IFAC-PapersOnLine, vol. 50, no. 1, pp. 3463-3469, 2017. 